\title{
COMPACTIFICATION BY THE TOPOLOGIST'S SINE CURVE
}

\author{
RONNIE LEVY
}

\begin{abstract}
Using a compactification of the nonnegative reals whose remainder is the topologist's sine curve, results about growths of Stone-Cech compactifications are proved. For example, it is proved that if $\beta X$ contains a nonconstant continuous image of a compact connected LOTS, then the image is contained in $v X$. This extends a result of Peter Nyikos.
\end{abstract}

In this note we discuss some of the consequences of the fact that the topologist's sine curve is $C R^{+}-R^{+}$for a particular compactification $C R^{+}$ of the nonnegative real numbers. In particular, we give a new proof of the fact that if $\beta X-X$ is path-connected, then $X$ is pseudocompact. We also give an apparently new proof of the fact, which was communicated in a letter by Peter Nyikos, that if $\beta X$ contains a nonconstant path then the path is contained in $v X$.

The author wishes to thank Peter Nyikos and the referee for many helpful suggestions which generalized the original versions of the theorems. In particular, in their original forms the results were stated for $T$-paths rather than locally connected continua.

1. Preliminaries. For general definitions, see [4]. All spaces are completely regular Hausdorff. If $X$ is any space, $\beta X$ denotes the Stone-Čech compactification of $X$ and $v X$ denotes the Hewitt realcompactification of $X$. A continuum is a compact connected space. $R$ denotes the set of real numbers and $R^{+}$denotes the set of nonnegative real numbers. The topologist's sine curve is the space $S=S_{1} \cup S_{2}$ where

$$
S_{1}=\{0\} \times[-1,1] \text { and } S_{2}=\{(x, \sin (1 / x)): 0<x \leqslant 1\} .
$$

$S$ is given the induced topology from $R^{2}$.

It is known that $S$ is the remainder of some compactification of $R^{+}$(see, for example, [4]). It is convenient for the sake of reference, however, to describe a particular compactification, which we call $C R^{+}$, such that $C R^{+}-$ $R^{+}$is $S$.

We observe that the minima of the function $\sin (1 / x)$ (defined on $(0,1])$ are at $x=2 /[(4 n-1) \pi]$ for $n=1,2, \ldots$, and the minimum value is -1 . For $n=1,2, \ldots$, let $B_{n}=\{(x, 1 / n): 2 /[(4 n-1) \pi] \leqslant x \leqslant 1\}$, so for each $n, B_{n}$

Received by the editors October 13, 1975 and, in revised form, June 1, 1976.

AMS (MOS) subject classifications (1970). Primary 54D40, 54F15.

Key words and phrases. $T$-path, Stone-Cech compactification.

(1) American Mathematical Society 1977 
is a horizontal line segment which is $1 / n$ units above the $x$-axis. Let $C_{n}$ be the line segment joining the left endpoint of $B_{n}$ to the right endpoint of $B_{n+1}$. Formally,

$$
C_{n}=\left\{\left(x, \frac{\pi(1-4 n)(x-1)}{n(n+1)[(4 n-1) \pi-2]}+\frac{1}{n+1}\right): \frac{2}{(4 n-1) \pi} \leqslant x \leqslant 1\right\} .
$$

Let $A=\cap_{n=1}^{\infty}\left(B_{n} \cup C_{n}\right)$. To obtain $C R^{+}$we "bend" $A$ in such a way that it conforms to $S$. Let $L=\{(x, \sin (1 / x))+(x, y):(x, y) \in A\} . L$ is clearly homeomorphic to $R^{+}$. Let $C R^{+}=S \cup L$. Then $C R^{+}$is clearly a compactification of $R^{+}$(where we view $R^{+}$as $L$ ) and $C R^{+}-L=S$. For $k=$ $1,2, \ldots$ let $p_{k}=(2 /[(4 k-1) \pi],(1 / k)-1)$; then the $p_{k}$ 's are the "sharp" points of $L$ (that is, the nondifferentiable points of $L$ ) whose $x$-coordinates are not 1 .

2. Path-connectedness properties of $\beta R^{+}$. For the remainder of this paper let $T=[a, b]$ be any compact connected linearly ordered topological space with $a<b$.

LEMMA 2.1. If $K$ is a locally connected continuum and $f: K \rightarrow S$ is continuous, then either $f(K) \subseteq S_{1}$ or $f(K) \subseteq S_{2}$.

Proof. Any locally connected continuum contained in $S$ is clearly contained in either $S_{1}$ or $S_{2}$, and local connectedness is preserved under closed continuous maps (see [2] for example).

THEOREM 2.2. If $K$ is a nontrivial continiuum contained in $\beta R^{+}-R^{+}$, then there is a continuous function $G: K \rightarrow S$ whose range meets both $S_{1}$ and $S_{2}$.

Proof. Suppose $p, q \in K, p \neq q$. Let $\tilde{\tilde{U}}$ and $\tilde{\tilde{V}}$ be disjoint closed $\beta R^{+}$. neighborhoods of $p$ and $q$ and let $U=\tilde{\tilde{U}} \cap R^{+}, V=\tilde{V} \cap R^{+}$. Choose an increasing cofinal sequence $a_{1}<a_{2}<\ldots$ in $R^{+}$such that

$$
a_{i} \in R^{+} \backslash(U \cup V) \text { for each } i \text {. }
$$

Define $g: U \cup V \rightarrow L$ by $g\left|\left[a_{n}, a_{n+1}\right] \cap U=(1,(\sin 1)+1), g\right|\left[a_{n}, a_{n+1}\right] \cap$ $V=p_{n}$. Since the sets $\left[a_{n}, a_{n+1}\right] \cap U$ and $\left[a_{n}, a_{n+1}\right] \cap V$ are disjoint and open in $U \cup V, g$ is well defined and continuous. $g$ extends continuously to $\tilde{\tilde{g}}: R^{+} \rightarrow L . \tilde{\tilde{g}}$ extends continuously to $\tilde{\tilde{g}}^{*}: \beta R^{+} \rightarrow C R^{+}$. Then $G=\tilde{\tilde{g}}^{*} \mid K$ is the required function.

Definition. If $X$ is a space and $f: T \rightarrow X$ is continuous, $f$ is called a $T$-path joining $f(a)$ to $f(b)$; its image, which is also called a $T$-path, is denoted $\tilde{\tilde{T}}$.

Combining Lemma 2.1 and Theorem 2.2 for the special case when $K$ is a $T$-path gives the following:

Corollary 2.3. (See also[1].) $\beta R^{+}-R^{+}$contains no nonconstant T-path.

Proposition 2.4. If $\beta R^{+}$contains a nontrivial locally connected continuum $K, K \subseteq R^{+}$. 
Proof. It suffices to show that there can be no pair $a, b \in K$ with $a \in R^{+}, b \in \beta R^{+}-R^{+}$. Suppose there were such a pair; let $g$ be a continuous map from $\beta R^{+}$to $S$ such that $g \mid R^{+}$is a homeomorphism onto $S_{2}$. Then $g(b) \in S_{1}, g(a) \in S_{2}$, contradicting 2.1 .

3. $T$-paths in $\beta X$. A special case of the following theorem was given in a letter by Peter Nyikos, but his proof was quite different.

THEOREM 3.1. If $X$ is realcompact and $f: T \rightarrow \beta X$ is a nonconstant $T$-path, then $\tilde{\tilde{T}} \subseteq X$.

Proof. Suppose $p \neq q, p, q \in \tilde{\tilde{T}}, p \in \beta X-X$. There is a $g \in C(\beta X)$ such that $0 \leqslant g \leqslant 1, g(q)=0, g(p)=1$. Since $X$ is realcompact, there is an $h \in C(X), h \geqslant 0$, such that $h$ does not extend to $p$. Let $F=(g \mid X)(h \mid X)$. Then $F$ extends to $q$ (or is already defined at $q$ ) but $F$ does not extend to $p$. We now view $F$ as a function from $X$ to $\beta R^{+}$and let $F^{*}: \beta X \rightarrow \beta R^{+}$be its Stone extension. Then $F^{*}(q)=0, F^{*}(p) \notin R^{+}$so $F^{*}$ is a nonconstant $T$-path containing points of $\beta R^{+}-R^{+}$, contradicting Corollary 2.3.

COROLlaRY 3.2. If $\tilde{\tilde{T}}$ is a nonconstant $T$-path in $\beta X$, then $\tilde{\tilde{T}} \subseteq v X$.

Proof. $\beta(v X)=\beta X$. Apply Theorem 3.1 to $v X$.

REMARK. We note that Theorem 3.1 and Corollary 3.2 hold with "nonconstant $T$-path" replaced with "nontrivial locally connected continuum."

Corollary 3.3. If $\beta X-X$ is path connected, or if $\beta X$ is path connected, or if every point of $\beta X-X$ is an element of some nonconstant $T$-path of $\beta X$ for some $T$, then $X$ is pseudocompact.

Proof. By Corollary 3.2, if every point of $\beta X-X$ is an element of a nonconstant $T$-path, then every point of $\beta X-X$ is in $v X$, that is $\beta X=v X$.

\section{REFERENCES}

1. D.P. Bellamy, A non-metric indecomposable continuum, Duke Math. J. 38 (1971), 15-10. MR 42 \# 6792.

2. J. Dugundji, Topology, Allyn and Bacon, Boston; 1966. MR33 \# 1824.

3. L. Gillman and M. Jerison, Rings of continuous functions, Van Nostrand, Princeton, N.J., 1960. MR22 \# 6994.

4. J.W. Rogers, Jr., On compactifications with continua as remainders, Fund. Math. 70 (1971), no. 1, 7-11. MR 44 \# 993.

Department of Mathematics, George Mason University, Fairfax, Virginia 22030 